\section{Doctors' non-verbal communication}

As stated in the February editorial, GPs 'now use computers extensively in their practice'.'

If doctors are to work on their computer, it would be beneficial if they were to master touch typing so that they can use their fingers on the keyboard without losing eye-contact with the patient. This does not abolish the problem of the lower body facing the computer although the use of a swivel chair would allow the doctor to face the patient at the beginning of the consultation and using the keyboard only when necessary.

\section{lain Esslemont,}

Eoisle Monadh, 2 Chardonnay Avenue, Margaret River, Western Australia 6285. E-mail: esslemont@wn.com.au

\section{REFERENCE}

1. Silverman J, Kinnersley P. Doctors' non-verbal behaviour in consultations: look at the patient before you look at the computer. Br J Gen Pract 2010: 60(571): 76-78.

DOI: 10.3399/bjgp10X509658

\section{Looking at the patient}

I read with interest the letter 'looking at the patient" published in the April BJGP, especially as I have been reflecting on my own consultations recently.

I, too, have learned to touch type so that I can record notes while listening to and looking at the patient (except when have to work in a room in the computer is angled in a different direction to the patient). I sometimes also 'speak as I write' so that patients are able to hear what I am recording, and can correct me if I erroneously type 'right' instead of 'left', for example. However, as a conscientious risk-averse doctor I find that I make longer notes than most of my GP colleagues, that has led to feedback that my notes are too long and take too much time for my colleagues to read. The length of my notes may be partly due to the fact that I do type quickly and can therefore record more data more quickly, but conversely I may be less discriminating in what I record.

Last year at my practice appraisal it was suggested that I try to type the data into the records at the end of the consultation rather than as it progressed, as this may help me to produce a more concise summary rather than the potentially rambling real-time recording Looking back, this 'summarising' approach may have coincided with a slight but significant reduction in my consultation length, bringing it nearer to the allotted 10 minutes than my previous over-run. Unfortunately, I did not manage to continue this improvement, but looking back it might have been this change of approach to 'summary recording' that had helped me quicken my appointment time, albeit not maintained.

I do take pride in my thorough notes, both as a medico-legal defence and indeed for good ongoing patient care, or if I were to be hit by the proverbial bus. Interestingly, I heard it reported that a medico-legal advisor stated that half of the consultation time should be used for note-making, and of course if it isn't recorded 'it didn't happen'. It is therefore hard for me to experiment with deliberately shortening my records that I take great pride in. However, it is something I am willing to try again, while still aiming to record a careful history with relevant positives and negatives, clear safety-netting advice, etc, in the context of high-quality, patient-centred, conscientious care. Actually while trying with this briefer approach again today I find that in some more complex presentations I feel compelled to record contemporaneously, perhaps haunted by a sleep-deprived junior house officer clerking, in that I hadn't recorded as I'd gone along and had to rely on the senior house officer kindly re-clerking the patient as l'd forgotten the details! The craft of perfecting the consultation, with its multitasking and varied demands, remains a challenging art that I continue to develop and review throughout my career.
Nicola D Jones,

Salaried Doctor on the Doctors' Retainer Scheme, Roseneath Medical Practice,

Buckley, CH7 2JL.

E-mail: nicolajones@doctors.org.uk

REFERENCE

1. Hay GI. Looking at the patient. Br J Gen Pract 2010; 60(573): 293

DOI: 10.3399/bjgp10X509667

\section{TV scheduling - a tool for public health?}

Good quality sleep of adequate duration is recognised as vital in maintaining both physical and psychological health. According to Alvarez and Ayas, ${ }^{1}$ a variety of studies have suggested an association between self-reported sleep duration and health. Those patients who report both a reduced ( $<7$ hours/day) or an increased (>8hours/day) sleep duration are at a modestly increased risk of all-cause mortality, coronary heart disease, and diabetes mellitus.

Research conducted by the Sleep Research Centre at Loughborough University, found that the most common time that adults in the UK fell asleep and woke up was 23:30 and 07:00 respectively, with an average sleep duration of 6.9 hours for men and 7.2 hours for women.

There are a variety of documented factors that impact on the length and quality of sleep including stress, some medical conditions, certain medications, what people eat and drink, and their 'sleep environment'. ${ }^{3}$ Another important factor is the increased time spent watching television. ${ }^{1}$ People in the UK spend an average of 3.6 hours per day watching TV, the majority of which is in the evening. ${ }^{4}$

I would like to argue that an overlooked factor that may affect the duration of sleep is TV scheduling. To look at how this could potentially affect sleep length, I decided to focus on the scheduling of movies that are some of the most popular 